\title{
A study of clinico-hematological profile of pancytopenic patients in Central India
}

\author{
Yadav $\mathbf{A}^{1}$, Nigam $\mathbf{R} \mathbf{K}^{2}$, Malik $\mathbf{R}^{3}$ \\ ${ }^{1}$ Dr. Amita Yadav, PG Resident, ${ }^{2}$ Dr. R.K. Nigam, Professor, ${ }^{3}$ Dr. Reeni Malik, Professor and Head of the Department, \\ all authors are affiliated with Department of Pathology, Gandhi Medical College, Bhopal, Madhya Pradesh, India.
}

Address for Correspondence: Dr. Amita Yadav, D/O Shri Bhola Nath Singh Yadav, S-26/42, PBN, Neerja Niwas, Ashokpuram Colony, Meerapur Basahi, Varanasi, Uttar Pradesh, India. Email: amita.ritu@gmail.com

\begin{abstract}
Background: To segregate the causes of pancytopenia which are easily treatable, from those requiring sophisticated investigations \& vigorous treatment, based on clinico-hematological profile of the patients and affordable diagnostic mathods. Materials and Methods: 180 Pancytopenic patients were evaluated clinically along with hematological parameters, peripheral smears and bone marrow aspiration in the Department of Pathology, GMC, Bhopal for 3 years. Result: Age range was 5 months-70 years (mean- 26.6 years), with Male preponderance (M: F: 1.76:1). Commonest symptoms were weakness $(97.8 \%)$, breathlessness $(75 \%)$ and signs were pallor $(98.3 \%)$ and splenomegaly $(25.5 \%)$. Patients who presented with per rectal bleeding, in $46.2 \%$ diagnosis was dimorphic anemia thus per rectal bleeding was cause of associated iron deficiency. Commonest peripheral smear finding was microcytic hypochromic picture (27.22\%) \& bone marrow was hypercellular $(70.00 \%)$. Bone marrow aspiration revealed commonest cause was megaloblastic anemia (25\%) followed by dimorphic anemia (17.2\%) and infections (17.2\%). In our study MCV was $<100 \mathrm{fl}$ in $12 / 45$ $(26.66 \%)$ cases of megaloblastic anemia so even if a patient presents with MCV $<100 \mathrm{fl}$ megaloblastic anemia should not be ruled out only on this basis. Sensitivity of peripheral smear for dimorphic anemia was very low $35.48 \%$ and specificity was $82.55 \%$, so bone marrow examination should be investigation of choice for inconclusive peripheral smears. Conclusion: Common causes of pancytopenia were easily treatable and reversible \& can be detected by early and affordable diagnostic methods therefore should be considered at higher order in differential diagnosis of pancytopenia.
\end{abstract}

Key words: Bone marrow aspiration, Megaloblastic anemia, Pancytopenia

\section{Introduction}

Pancytopenia is the simultaneous presence of anemia, leucopenia and thrombocytopenia. Pancytopenia therefore exists in the adult when the hemoglobin level is less than $13.5 \mathrm{gm} / \mathrm{dl}$ in males or $11.5 \mathrm{gm} / \mathrm{dl}$ in females, the leucocyte count is less than $4 \times 10^{9} / \mathrm{L}$ and the platelet count is less than $150 \times 10^{9} / \mathrm{L}[1]$.

Although all the cell-lines of the blood are depleted, the clinical manifestations are mutually different from one another due to various causes of pancytopenia. The etiology of pancytopenia varies in different populations depending upon age, prevalence of infections,

Manuscript received: $6^{\text {th }}$ May 2017

Reviewed: $16^{\text {th }}$ May 2017

Author Corrected: $24^{\text {th }}$ May 2017

Accepted for Publication: $31^{\text {st }}$ May 2017 nutritional status and environment. So this study is conducted to evaluate the clinical profile, etiological spectrum and, peripheral blood \& bone marrow findings of pancytopenic patients, with the aim to segregate easily treatable and reversible causes of pancytopenia from the diseases which need vigorous treatment in the central part (Bhopal region of Madhya Pradesh) of India, as there is paucity of authentic data about this.

Bone marrow aspiration, relatively safe and easy test, except for a mild discomfort to the patients, plays important role in determining the cause for pancytopenia, unexplained cytopenias, storage disorders and hematological malignancies [2]. As our hospital is a tertiary care centre, most of the patients belong to 
nearby rural areas, have poor socioeconomic status and they are unable to afford costly investigations. Thus there is a need to identify a pattern in the symptoms so as to pick up the clues to find out the exact etiology. A correlation between the clinical features of individual patients and the blood picture, bone marrow examination is being reviewed in this study in order to achieve the same. Such a correlation will help the clinicians to reach at the diagnosis easily and early. So this study is conducted to find out the causes of pancytopenia at earliest with minimal economical resources. Importance of the study lies in the timely intervention for the etiology of pancytopenia which can either bring about a complete cure or at least a remission from the disease.

\section{Materials and Methods}

Patients with pancytopenia underwent a detailed history, clinical examination, complete Blood Count, peripheral smear and bone marrow aspiration as per proforma specially designated for this study. Assessment of hematological parameters was done by automated cell counter (Mindray BC 5300). Peripheral smears were prepared and stained by Leishman stain for all the cases and examined in detail. Bone marrow aspiration was subsequently performed under aseptic precaution using Salah's bone marrow aspiration needle after obtaining written consent from the patient or guardian. Bone marrow aspiration smears were also stained by Leishman stain and Perl's staining was done for assessment of iron status.
Study Design- Retrospective and prospective observational study.

Setting- Department of Pathology, Gandhi Medical College, Bhopal during a period of $1^{\text {st }}$ August 2013 to $31^{\text {st }}$ August 2016.

Inclusion Criteria- The study included all cases of pancytopenia i.e. hemoglobin less than $13.5 \mathrm{gm} / \mathrm{dl}$ in males or $11.5 \mathrm{gm} / \mathrm{dl}$ in females, total leucocyte count less than 4,000 cells/ cumm and platelet count less than $1,50,000 / \mathrm{cu} \mathrm{mm}$.

Exclusion Criteria- Patients who had received chemotherapy, radiotherapy or immunosuppressive drugs were excluded from the study.

Participants- Patients diagnosed with pancytopenia and referred to our department for further work up.

Data Source- Data was collected from history, clinical examination, case files as well as investigations done in these patients.

Bias- Referral Bias.

Study size- This Study included 180 cases of pancytopenia.

Statistical Methods- For statistical analysis Chi square test was applied. $\mathrm{P}$ value was considered significant if $\mathrm{p}<0.05$, and highly significant if $\mathrm{p}<0.01$. Percentage, mean, standard deviation, sensitivity and specificity were also calculated.

\section{Results}

Table-1: Age group wise distribution of cases according to final diagnosis.

\begin{tabular}{|c|c|c|c|c|}
\hline Final Diagnosis & $\begin{array}{c}\mathbf{0 - 1 4} \text { years } \\
(\mathbf{n = 4 8})\end{array}$ & $\begin{array}{c}\mathbf{1 5 - 3 4 y e a r s} \\
(\mathbf{n = 7 2})\end{array}$ & $\begin{array}{c}\text { >34years } \\
(\mathbf{n = 6 0})\end{array}$ & Total \\
\hline Megaloblastic Anemia & $9(5 \%)$ & $18(10 \%)$ & $18(10 \%)$ & $45(25 \%)$ \\
\hline Dimorphic Anemia & $8(4.4 \%)$ & $13(7.2 \%)$ & $10(5.6 \%)$ & $31(17.2 \%)$ \\
\hline Marrow Reactive to infection & $6(3.3 \%)$ & $14(7.7 \%)$ & $11(6.2 \%)$ & $31(17.2 \%)$ \\
\hline Aplastic/ Hypoplasic Anemia & $8(4.4 \%)$ & $11(6.1 \%)$ & $4(2.3 \%)$ & $23(12.8 \%)$ \\
\hline Acute Myeloid Leukemia & $5(2.8 \%)$ & $6(3.3 \%)$ & $6(3.3 \%)$ & $17(9.4 \%)$ \\
\hline Acute Lymphoblastic Leukemia & $4(2.3 \%)$ & $3(1.6 \%)$ & $1(0.6 \%)$ & $8(4.4 \%)$ \\
\hline Trilineage Hyperplasia with & $1(0.6 \%)$ & $3(1.6 \%)$ & $3(1.6 \%)$ & $7(3.8 \%)$ \\
\hline hypersplenism & & & & $3(1.6 \%)$ \\
\hline Lymphoma & $0(\%)$ & $1(0.6 \%)$ & $1(0.6 \%)$ & $3(1.6 \%)$ \\
\hline Plasma Cell Dyscrasia & $1(0.6 \%)$ & $1(0.6 \%)$ & $1(0.6 \%)$ & $3(1.6 \%)$ \\
\hline Gaucher's Disease & $2(1.1 \%)$ & $0(0 \%)$ & $2(1.1 \%)$ & $8(4.4 \%)$ \\
\hline Others & $4(2.2 \%)$ & $2(1.1 \%)$ & $60(33.5 \%)$ & $180(100 \%)$ \\
\hline Total & $48(26.7 \%)$ & $72(39.8 \%)$ & & $4.6 \%)$ \\
\hline
\end{tabular}


Most common cause of pancytopenia found was megaloblastic anemia (25\%), followed by dimorphic anemia (17.2\%) and bone marrow reactive to infection (17.2\%). Age range was 5 months to 70 years (Mean-26.6 years). Males were $63.8 \%$ and females were 36.2\%. A definite male preponderance (Male: Females: 1.76:1) was observed. Male: female ratio in pediatric age group was slightly higher (2.69:1). Most common age group (39.8\%) was 15-34 years. In all the age groups most common cause of pancytopenia was megaloblastic anemia. (Table-1).

Table 2-Gender wise distribution of symptoms.

\begin{tabular}{|c|c|c|c|c|}
\hline Symptoms & Males No. (\%) & Females No. (\%) & Total No. (\%) & P value \\
\hline Weakness & $113(62.8 \%)$ & $63(35.0 \%)$ & $176(97.8 \%)$ & 0.558 \\
\hline Breathlessness & $93(51.7 \%)$ & $42(23.3 \%)$ & $135(75 \%)$ & $0.015 * *$ \\
\hline Fever & $83(46.2 \%)$ & $43(23.8 \%)$ & $126(70 \%)$ & 0.397 \\
\hline Epistaxis & $20(11.2 \%)$ & $8(4.4 \%)$ & $28(15.6 \%)$ & 0.366 \\
\hline Hematemesis & $7(3.9 \%)$ & $9(5.0 \%)$ & $16(8.9 \%)$ & 0.078 \\
\hline Per rectal bleeding & $12(6.7 \%)$ & $1(0.5 \%)$ & $13(7.2 \%)$ & $0.026^{* *}$ \\
\hline Gum bleeding & $7(3.9 \%)$ & $3(1.7 \%)$ & $10(5.6 \%)$ & 0.678 \\
\hline Ecchymosis & $6(3.4 \%)$ & $3(1.6 \%)$ & $9(5.0 \%)$ & 0.858 \\
\hline Rashes & $7(3.9 \%)$ & $2(1.1 \%)$ & $9(5.0 \%)$ & 0.373 \\
\hline Petechiae & $5(2.7 \%)$ & $3(1.6 \%)$ & $8(4.4 \%)$ & 0.933 \\
\hline Hematuria & $0(0 \%)$ & $2(1.1 \%)$ & $2(1.1 \%)$ & NA \\
\hline Pedal edema & $9(5.0 \%)$ & $6(3.3 \%)$ & $15(8.3 \%)$ & 0.743 \\
\hline
\end{tabular}

** Statistically significant as $\mathrm{p}<0.05$

NA- No. of cases were $<5$, so chi square test was not applied.

Most common symptom was generalized weakness (97.8\%) followed by breathlessness (75\%), fever (70\%) and bleeding tendencies. Most common bleeding tendency was epistaxis (15.6\%) followed by hematemesis $(8.9 \%)$, per-rectal bleeding $(7.2 \%)$ and others. Breathlessness $(p=0.015)$ and per rectal bleeding $(p=0.026)$ were more significant in males as compared to females. (Table-2).

13/180 patients presented with per rectal bleeding and 6/13 (46.2\%) were diagnosed as case of Dimorphic anemia thus, per rectal bleeding is a cause of associated iron deficiency.

Table-3: Gender wise distribution of clinical signs.

\begin{tabular}{|c|c|c|c|c|}
\hline Signs & Males No. (\%) & Females No. (\%) & Total No. (\%) & P value \\
\hline Pallor & $112(62.2 \%)$ & $65(36.1 \%)$ & $177(98.3 \%)$ & 0.189 \\
\hline Splenomegaly & $29(16.1 \%)$ & $17(9.4 \%)$ & $46(25.5 \%)$ & 0.889 \\
\hline Hepatomegaly & $24(13.3 \%)$ & $9(5.0 \%)$ & $33(18.3 \%)$ & 0.242 \\
\hline Icterus & $20(11.2 \%)$ & $12(6.6 \%)$ & $32(17.8 \%)$ & 0.856 \\
\hline Lymphadenopathy & $20(11.1 \%)$ & $3(1.6 \%)$ & $23(12.7 \%)$ & $0.013 * *$ \\
\hline Ascites & $6(3.3 \%)$ & $9(5.0 \%)$ & $15(8.3 \%)$ & $0.044 * *$ \\
\hline
\end{tabular}

**Statistically significant as $\mathrm{p}<0.05$

NA- No. of cases were $<5$, so chi square test was not applied.

Most common sign was pallor (98.3\%) followed by splenomegaly $(25.5 \%)$, hepatomegaly (18.3\%) and others. Lmphadenopathy $(\mathrm{p}=0.013)$ was more significant in males and ascites $(\mathrm{p}=0.044)$ was more significant in females. In present study among 8 cases of ALL, one patient (12.5\%) presented without hepatosplenomegaly and lymphadenopathy (Aplastic Presentation). 
Table-4: Comparison of hematological parameters among leading causes of pancytopenia.

\begin{tabular}{|c|c|c|c|c|}
\hline Diagnosis & $\begin{array}{c}\text { Hemoglobin } \\
\text { Mean } \pm \text { SD }\end{array}$ & $\begin{array}{c}\text { Total RBC Count } \\
\text { Mean } \pm \text { SD }\end{array}$ & $\begin{array}{c}\text { Total WBC } \\
\text { Count } \\
\text { Mean } \pm \text { SD }\end{array}$ & $\begin{array}{c}\text { Platelet Count } \\
\text { Mean } \pm \text { SD }\end{array}$ \\
\hline Megaloblastic Anemia & $5.57 \pm 1.93 \mathrm{gm} / \mathrm{dl}$ & $\begin{array}{c}2.15 \pm 0.84 \times 10^{6} \\
\text { /cumm) }\end{array}$ & $\begin{array}{c}2700 \pm 853 \\
\text { cells/cumm) }\end{array}$ & $\begin{array}{c}47000 \pm 28,000 \\
\text { /cumm) }\end{array}$ \\
\hline Dimorphic Anemia & $5.69 \pm 1.95 \mathrm{gm} / \mathrm{dl}$ & $\begin{array}{c}2.62 \pm 0.84 \times 10^{6} \\
/ \text { cumm }\end{array}$ & $\begin{array}{c}3000 \pm 800 \\
\text { cells/cumm) }\end{array}$ & $\begin{array}{c}66,000 \pm 28,000 \\
\text { /cumm) }\end{array}$ \\
\hline $\begin{array}{c}\text { Marrow Reactive to } \\
\text { infection }\end{array}$ & $\begin{array}{c}6.19 \pm 1.93 \\
\mathrm{gm} / \mathrm{dl}\end{array}$ & $\begin{array}{c}2.55 \pm 0.84 \times 10^{6} \\
\text { /cumm }\end{array}$ & $\begin{array}{c}3000 \pm 800 \\
\text { Cells/cumm) }\end{array}$ & $\begin{array}{c}49000 \pm 28,000 \\
\text { /cumm) }\end{array}$ \\
\hline $\begin{array}{c}\text { Aplastic/ Hypoplasic } \\
\text { Anemia }\end{array}$ & $\begin{array}{c}4.73 \pm 1.94 \\
\mathrm{gm} / \mathrm{dl}\end{array}$ & $\begin{array}{c}1.21 \pm 0.84 \times 10^{6} \\
\text { /cumm }\end{array}$ & $\begin{array}{c}2300 \pm 800 \\
\text { Cells/cumm) }\end{array}$ & $\begin{array}{c}40000 \pm 28,000 \\
\text { /cumm) }\end{array}$ \\
\hline Acute Myeloid Leukemia & $\begin{array}{c}5.58 \pm 1.95 \\
\mathrm{gm} / \mathrm{dl}\end{array}$ & $\begin{array}{c}1.98 \pm 0.84 \times 10^{6} \\
\text { /cumm }\end{array}$ & $\begin{array}{c}2700 \pm 800 \\
\text { cells/cumm) }\end{array}$ & $\begin{array}{c}36000 \pm 28,000 \\
\text { cumm) }\end{array}$ \\
\hline $\begin{array}{c}\text { Acute Lymphoblastic } \\
\text { leukemia }\end{array}$ & $5.28 \pm 1.94 \mathrm{gm} / \mathrm{dl}$ & $\begin{array}{c}2.18 \pm 0.84 \times 10^{6} \\
\text { /cumm }\end{array}$ & $\begin{array}{c}2600 \pm 800 \\
\text { Cells/cumm) }\end{array}$ & $\begin{array}{c}48000 \pm \\
28,000 / \text { cumm }\end{array}$ \\
\hline $\begin{array}{c}\text { Trilineage Hyperplasia } \\
\text { with hypersplenism }\end{array}$ & $\begin{array}{c}6.42 \pm 1.92 \\
\mathrm{gm} / \mathrm{dl}\end{array}$ & $\begin{array}{c}2.60 \pm 0.77 \times 10^{6} \\
\text { /cumm }\end{array}$ & $\begin{array}{l}2500 \pm 800 \\
\text { Cells/cumm }\end{array}$ & $\begin{array}{c}59000 \pm 28,000 \\
\text { /cumm }\end{array}$ \\
\hline
\end{tabular}

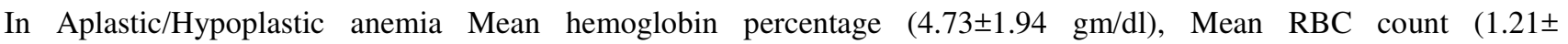
$\left.0.84 \times 10^{6} / \mathrm{cumm}\right)$ and mean total leucocyte count $(2300 \pm 800$ cells/cumm) were lowest in comparison to other causes of pancytopenia.

Table-5: Diagnosis wise MCV values among leading causes of pancytopenia.

\begin{tabular}{|c|c|c|c|c|}
\hline & \multicolumn{3}{|c|}{ MCV } & \multirow[b]{2}{*}{$\begin{array}{c}\text { Total } \\
\operatorname{cases}(\mathbf{n})\end{array}$} \\
\hline Diagnosis & $<80$ fl & 80-100 fl & $>100 \mathrm{fl}$ & \\
\hline Megaloblastic Anemia & $4(8.9 \%)$ & $8(17.77 \%)$ & $33(73.33 \%)$ & $45(100 \%)$ \\
\hline Dimorphic Anemia & $15(48.38 \%)$ & $12(38.7 \%)$ & $4(12.92 \%)$ & $31(100 \%)$ \\
\hline Marrow Reactive to infection & $14(45.17 \%)$ & $15(48.38 \%)$ & $2(6.45 \%)$ & $31(100 \%)$ \\
\hline Aplastic/ Hypoplasic Anemia & $6(26.08 \%)$ & $16(69.56 \%)$ & $1(4.36 \%)$ & $23(100 \%)$ \\
\hline Acute Myeloid Leukemia & $3(17.66 \%)$ & $10(58.82 \%)$ & $4(23.52 \%)$ & $17(100 \%)$ \\
\hline Acute Lymphoblastic Leukemia & $3(25 \%)$ & $7(58.3 \%)$ & $2(16.7 \%))$ & $12(100 \%)$ \\
\hline $\begin{array}{c}\text { Trilineage Hyperplasia with } \\
\text { hypersplenism }\end{array}$ & $6(85.71 \%)$ & $1(14.29 \%)$ & $0(0 \%)$ & $7(100 \%)$ \\
\hline
\end{tabular}

$12 / 45(26.66 \%)$ cases of megaloblastic anemia presented with MCV values $<100 \mathrm{fl}$. So even if a patient presents with MCV value of $<100 \mathrm{fl}$, megaloblastic anemia should not be ruled out only on this basis.

Table-6: Comparison of organomegaly in cases of cellular and hypocellular marrow.

\begin{tabular}{|c|c|c|c|c|}
\hline & $\begin{array}{c}\text { Hypercellular }+ \\
\text { Normocellular }\end{array}$ & Hypocellular & Total & P value \\
\hline Hepatomegaly & $28(84.8 \%)$ & $5(15.2 \%)$ & $33(100 \%)$ & 0.279642. \\
\hline Splenomegaly & $42(91.3 \%)$ & $4(8.7 \%)$ & $46(100 \%)$ & $0.01054 * *$ \\
\hline
\end{tabular}

*** Statistically significant as $\mathrm{p}<0.05$

Bone marrow was hypercellular in $70.00 \%$ cases followed by hypocellular marrow $(22.22 \%)$ and normocellular marrow (7.78\%). We also found association of organomegaly with bone marrow cellularity. There was significant association of splenomegaly with cellular marrow $(\mathrm{p}=0.010)$. 
Peripheral smear findings- In peripheral smear anisopoikilocytosis was predominant finding. Microcytic hypochromic RBCs were seen in $27.22 \%$ cases followed by normocytic hypochromic RBCs (26.67\%), Macrocytic picture were seen in $25 \%$ cases and dimorphic picture was seen in $20.56 \%$ cases.

Table-7: Sensitivity and specificity of Peripheral smear examination as compared to bone marrow examination

A. Megaloblastic Anemia.

\begin{tabular}{|c|c|c|c|}
\hline Peripheral smear & $\begin{array}{c}\text { Final Diagnosis by bone marrow } \\
\text {-Megaloblastic Anemia ( + ) }\end{array}$ & $\begin{array}{c}\text { Final Diagnosis by bone marrow- } \\
\text { Megaloblastic Anemia ( - ) }\end{array}$ & Total \\
\hline Macrocytic picture( +) & 31 & 14 & 45 \\
\hline Macrocytic picture( - ) & 14 & 121 & 135 \\
\hline Total & 45 & 135 & 180 \\
\hline
\end{tabular}

- Sensitivity- $68.89 \%$

- Specificity- $89.63 \%$

B. Dimorphic Anemia-

\begin{tabular}{|c|c|c|c|}
\hline Peripheral smear & $\begin{array}{c}\text { Final Diagnosis by bone } \\
\text { marrow-Dimorphic Anemia } \\
(+)\end{array}$ & $\begin{array}{c}\text { Final Diagnosis by bone } \\
\text { marrow- Dimorphic Anemia } \\
(-)\end{array}$ & Total \\
\hline Dimorphic picture( +) & 11 & 26 & 37 \\
\hline Dimorphic picture( - ) & 20 & 123 & 143 \\
\hline Total & 31 & 149 & 180 \\
\hline
\end{tabular}

- Sensitivity- $35.48 \%$

- Specificity- $82.55 \%$

Sensitivity of peripheral smear for diagnosis of megaloblastic anemia was $68.89 \%$ and specificity was $89.63 \%$ whereas for dimorphic anemia sensitivity was very low $35.48 \%$ and specificity was $82.55 \%$ (bone marrow aspiration was taken as gold standard test) so bone marrow examination should be investigation of choice in case of inconclusive peripheral smears.

\section{Discussion}

In our study megaloblastic anemia (25\%) was most common cause of pancytopenia. Tilak \& Jain et al. reported higher incidence of megaloblastic anemia $68 \%$. Jha et al. found $23.64 \%$ and Bhatnagar et al. found $28.4 \%$ cases of megaloblastic anemia in their studies. [3, 10,11]

In pediatric age group also most common cause was megaloblastic anemia (18.75\%). Similar result was seen by Bhatnagar et al., they also reported megaloblastic anemia (28.4\%) as most common cause. In contrast Gupta et al. found aplastic anemia (43\%) as most common cause in children. [11, 13]

Very few studies have reported Dimorphic Anemia to be a major cause of pancytopenia. Dimorphic anemia (17.2\%) was second most common cause of pancytopenia in present study. Raphael et al. found $8.7 \%$ cases of dimorphic anemia as second most common cause and Prabhala et al. Found 14.53\% cases of dimorphic anemia as third most common cause of pancytopenia in their study. [8, 14].

A possible explanation of such large number of patients presenting with nutritional anemia in our study can be extremes of age, nutritional deficiencies, malabsorption, parasitic infestations, chronic gastrointestinal, genitourinary bleed etc. Since nutritional disorders are easily treatable therefore should be considered at higher order in differential diagnosis list of pancytopenia. 
Table-8: Comparison with other studies.

\begin{tabular}{|c|c|c|c|c|c|c|c|c|}
\hline Study & Country & Year & $\begin{array}{l}\text { No. of } \\
\text { cases }\end{array}$ & $\begin{array}{c}\text { Age } \\
\text { group }\end{array}$ & $\begin{array}{l}\text { M:F } \\
\text { ratio }\end{array}$ & $\begin{array}{c}\text { Commonest } \\
\text { cause }\end{array}$ & $\begin{array}{c}\text { 2nd common } \\
\text { cause }\end{array}$ & $\begin{array}{c}\text { 3rd common } \\
\text { cause }\end{array}$ \\
\hline $\begin{array}{c}\text { Tilak and Jain } \\
\text { et al. [3] }\end{array}$ & $\begin{array}{c}\text { India- } \\
\text { Chandigarh }\end{array}$ & 1999 & 77 & $5-70$ & $1.1: 1$ & $\begin{array}{c}\text { Megaloblasti } \\
\text { c Anemia } \\
(68 \%)\end{array}$ & $\begin{array}{c}\text { Aplastic } \\
\text { Anemia }(7.7 \%)\end{array}$ & $\begin{array}{c}\text { Other causes } \\
(24.3 \%)\end{array}$ \\
\hline $\begin{array}{c}\text { Kumar et } \\
\text { al. [4] }\end{array}$ & $\begin{array}{l}\text { India- } \\
\text { Delhi }\end{array}$ & 2001 & 166 & $12-73$ & $2.1: 1$ & $\begin{array}{l}\text { Aplastic } \\
\text { Anemia } \\
(29.5 \%)\end{array}$ & $\begin{array}{c}\text { Megaloblastic } \\
\text { Anemia } \\
(22.3 \%)\end{array}$ & $\begin{array}{c}\text { Aleukemic } \\
\text { leukemia } \\
(12 \%\end{array}$ \\
\hline $\begin{array}{l}\text { Hamid et } \\
\text { al. [5] }\end{array}$ & Yemen & 2008 & 75 & $3-85$ & 1.03: 1 & $\begin{array}{c}\text { Hypersplenism } \\
(45.3 \%)\end{array}$ & $\begin{array}{c}\text { Megaloblastic } \\
\text { Anemia } \\
(14.7 \%)\end{array}$ & $\begin{array}{l}\text { Aplastic } \\
\text { Anemia } \\
(13.3 \%)\end{array}$ \\
\hline $\begin{array}{c}\text { Santra \& Das } \\
\text { et al. [6] }\end{array}$ & $\begin{array}{l}\text { India- } \\
\text { Kolkata }\end{array}$ & 2010 & 111 & $13-65$ & $1.5: 1$ & $\begin{array}{c}\text { Aplastic } \\
\text { Anemia } \\
(22.72 \%)\end{array}$ & $\begin{array}{c}\text { Hypersplenism } \\
(15 \%)\end{array}$ & $\begin{array}{c}\text { Drug induced } \\
(13 \%)\end{array}$ \\
\hline $\begin{array}{c}\text { Gayathri \& } \\
\text { Rao et al. [7] }\end{array}$ & $\begin{array}{c}\text { India- } \\
\text { Karnataka }\end{array}$ & 2011 & 104 & $2-80$ & $1.2: 1$ & $\begin{array}{c}\text { Megaloblastic } \\
\text { Anemia } \\
(74.04 \%)\end{array}$ & $\begin{array}{l}\text { Aplastic } \\
\text { Anemia } \\
(18.3 \%)\end{array}$ & $\begin{array}{c}\text { Subleukemic } \\
\text { Leukemia } \\
(3.8 \%)\end{array}$ \\
\hline $\begin{array}{l}\text { Raphael et } \\
\text { al. [8] }\end{array}$ & $\begin{array}{c}\text { India- } \\
\text { Meghalaya }\end{array}$ & 2012 & 80 & $1-79$ & $1: 1.2$ & $\begin{array}{c}\text { Megaloblastic } \\
\text { Anemia } \\
(41.2 \%)\end{array}$ & \begin{tabular}{|l|}
\multicolumn{1}{|c|}{ Dimorphic } \\
Anemia $(8.7 \%)$, \\
Aplastic Anemia \\
/ Hypoplastic \\
Anemia $(8.7 \%)$ \\
\end{tabular} & $\begin{array}{c}\text { Acute } \\
\text { Leukemia } \\
(7.5 \%)\end{array}$ \\
\hline $\begin{array}{c}\text { Jain and } \\
\text { Naniwadek } \\
\text { ar [9] }\end{array}$ & $\begin{array}{c}\text { India- } \\
\text { Maharashtra }\end{array}$ & 2013 & 250 & All & $2.6: 1$ & $\begin{array}{c}\text { Hypersplenism } \\
(29.2 \%)\end{array}$ & $\begin{array}{c}\text { Infections } \\
(25.6 \%)\end{array}$ & $\begin{array}{c}\text { Myelosuppre } \\
\text { ssants } \\
(16.8 \%)\end{array}$ \\
\hline $\begin{array}{l}\text { Present } \\
\text { Study }\end{array}$ & $\begin{array}{c}\text { India } \\
\text { - central } \\
\text { India }\end{array}$ & 2016 & 180 & $\begin{array}{c}5 \\
\text { months } \\
- \\
\text { 70years }\end{array}$ & $1.76: 1$ & $\begin{array}{l}\text { Megaloblastic } \\
\text { anemia }(25 \%)\end{array}$ & $\begin{array}{l}\text { Dimorphic } \\
\text { anemia and } \\
\text { Bone Marrow } \\
\text { reactive to } \\
\text { infection } \\
(17.2 \% \text { each })\end{array}$ & $\begin{array}{c}\text { Aplastic } \\
\text { /Hypoplastic } \\
\text { Anemia } \\
(12.8 \%)\end{array}$ \\
\hline
\end{tabular}

Bone marrow reactive to infections (17.2\%) was also second most common cause of pancytopenia in present study. Our finding is similar to Jain \& Naniwadekar et al. also found infective etiology (25.6\%) to be 2nd most common cause of pancytopenia in their study. Gupta et al. concluded infection (19.1\%) to be third most common cause of pancytopenia. Pine \& Walter et al. found infectious etiology (64\%) to be most common cause of pancytopenia $[9,13,15]$.

Infective etiology being a major cause of pancytopenia points towards poverty and poor hygienic conditions as our hospital is a tertiary care centre and majority of our patients belong to poor socioeconomic status. Aplastic anemia/hypoplastic anemia (12.8\%) was third most common cause of pancytopenia in our study. Similar result were seen in studies of Khodke et al. and Khunger et al., both observed an incidence of $14 \%$ and it was second most common cause of pancytopenia in their study [12, 16]. In contrast a higher incidence was reported by Kumar R et al. 29.5\%, Jha et al. found $29 \%$ and Verma N \& Dash S et al. reported $40.6 \%$ cases of aplastic anemia. Higher incidence of Aplastic / Hpoplastic anemia in other studies may be related to environmental factors such as increased exposure to toxic chemicals in industrialized areas, pesticide exposure and overuse and easy availability of over the counter drugs. [4, 10, 17].

In present study there was $13.8 \%$ incidence of Aleumic Leukemia (9.4\% incidence of Acute Myeloblastic Leukemia and $4.4 \%$ incidence of Acute Lymphoblastic Leukemia) compared to $12 \%$ reported by Kumar $R$ et al. and Khunger JM et al. reported $5 \%$ incidence of aleukemic leukemia $[4,16]$. 
Most common symptom was generalised weakness (97.8\%) followed by breathlessness (75\%), fever (70\%) and bleeding tendencies -epistaxis (15.6\%) followed by hematemesis $(8.9 \%)$, per-rectal bleeding (7.2\%), gum bleeding $(5.6 \%)$, ecchymosis(5.0\%), rashes(5.0\%) and petechiae(4.4\%). Santra \& Das et al. found weakness in 45\%, fever in $50.4 \%$ and bleeding tendencies in $41.4 \%$ cases. Khodke et al. found was fever in $40 \%$ followed by weakness in $30 \%$ and bleeding manifestations in $20 \%$ of the cases $[6,12]$.

Most common sign was pallor, which was present in $98.3 \%$ of cases followed by splenomegaly (25.5\%), hepatomegaly $(18.3 \%)$, icterus (17.8\%), lymphadenopathy (10.6\%) and ascites (8.3\%). Lmphadenopathy was more significant in males and ascites was more significant in females $(\mathrm{p}<0.05)$. Similarly Santra et al. found pallor in $84.6 \%$, hepatomegaly in $24 \%$ and splenomegaly in $44 \%$ cases. Khodke et al. reported pallor was universally present in all the patients, splenomegaly was seen in $40 \%$ and hepatomegaly in $38 \%$ of cases $[6,12]$.

Among hematological parameters in present study in Aplastic/Hypoplastic anemia Mean hemoglobin percentage (4.73 $\mathrm{gm} / \mathrm{dl})$, Mean RBC count $(1.21 \times 106 / \mathrm{cumm})$ and mean Total leucocyte count (2300cells/cumm) were lowest in comparison to other causes of pancytopenia. Azad et al. found in their study that mean Total leucocyte count (1600cells/cumm and platelets (25000/cumm) were lowest among patients of aplastic anemia [18]. 12/45(26.66\%) cases of megaloblastic anemia presented with MCV values $<100 \mathrm{fl}$. So even if a patient presents with MCV value of $<100 \mathrm{fl}$, megaloblastic anemia should not be ruled out only on this basis.

In present study in peripheral smear anisopoikilocytosis was predominant finding. Microcytic hypochromic RBCs were seen in $27.22 \%$ cases followed by normocytic hypochromic RBCs (26.67\%), Macrocytic picture were seen in $25 \%$ cases and Dimorphic picture was seen in $20.56 \%$ cases. Gayathri \& Rao et al. found in their study, the predominant blood picture was dimorphic anemia (37.5\%), followed by macrocytic anemia (31.7\%). Khodke et al. also found most common finding in peripheral blood film was dimorphic anaemia $(20 \%)$. [7, 12].

In present study bone marrow was hypercellular in $70.00 \%$ cases followed by hypocellular marrow (22.22\%) and normocellular marrow (7.78\%). Imbert $\mathrm{M}$ et al. found normo or hypercellular bone marrow in $66 \%$ cases. [19].

Present study shows significant association of splenomegaly with cellular marrow $(\mathrm{p}=0.010)$. Santra $\mathrm{G}$ et al. found hepatomegaly and splenomegaly both were significantly more common in patients with cellular marrow $(p<0.05)[6]$.

Thus present study stresses the importance of physical and peripheral blood findings in the management of pancytopenic patients. Sensitivity of peripheral smear examination was $68.89 \%$ and specificity was $89.63 \%$ for megaloblastic anemia. Sensitivity of peripheral smear for diagnosis of dimorphic anemia was very low (35.48\%) and specificity was $82.55 \%$, so bone marrow examination should be investigation of choice in case of inconclusive peripheral smears.

\section{Conclusion}

Our study reflects higher prevalence of nutritional anemia and infections in central part of India.These causes are easily treatable and reversible \& detected by early and affordable diagnostic methods therefore should be considered at higher order in differential diagnosis of pancytopenia. Present study stresses the importance of association of physical and peripheral blood findings in the management of pancytopenic patients.

Thus sophisticated $\&$ costly investigations and vigorous treatment, which may not be accessible everywhere and also not mandatory for all pancytopenic patients, especially in underdeveloped and developing countries, so there is need to conduct more studies on pancytopenia with larger cohort in future, which will definitely be helpful to decrease cost particularly for individuals, society and overall for countries with constraint resources like our India.

\author{
Abbreviations \\ Concentration. \\ Funding: Nil, Conflict of interest: None \\ Permission of IRB: Yes
}

Hb Haemoglobin, TLC Total Leucocyte Count, MCV Mean Corpuscular Volume, $\mathrm{MCH}$ Mean Corpuscular Hemoglobin, MCHC Mean Corpuscular Hemoglobin 


\section{References}

1. Lilleyman J. De Gruchy's Clinical Haematology in Medical Practice. J Clin Pathol. 1990 Apr;43 (4):352.

2. Jella R, Jella V. Clinico-hematological analysis of pancytopenia. Int J Adv Med. 2016;176-9.

3. Tilak V, Jain R. Pancytopenia--a clinicohematologic analysis of 77 cases. Indian J Pathol Microbiol. 1999 Oct 1;42(4):399.

4. Kumar R, Kalra SP, Kumar H, Anand AC, Madan H. Pancytopenia--a six year study. J Assoc Physicians India. 2001 Nov;49:1078-81.

5. Hamid GA, Shukry SAR. Patterns of pancytopenia in Yemen. J Turk Soc Haematol. 2008 Jun 5; 25 (2):71-4.

6. Santra G, Das BK. A cross-sectional study of the clinical profile and aetiological spectrum of pancytopenia in a tertiary care centre. Singapore Med J. 2010 Oct;51(10):806-12.

7. Gayathri BN, Rao KS. Pancytopenia: A Clinico Hematological Study. J Lab Physicians. 2011; 3(1):15-20.

8. Raphael V, Khonglah Y, Dey B, Gogoi P, Bhuyan A. Pancytopenia: an etiological profile. Turk J Haematol Off J Turk Soc Haematol. 2012 Mar; 29(1):80-1.

9. Jain A, Naniwadekar M. An etiological reappraisal of pancytopenia - largest series reported to date from a single tertiary care teaching hospital. BMC Blood Disord. 2013;13:10.

10. Jha A, Sayami G, Adhikari RC, Panta AD, Jha R. Bone marrow examination in cases of pancytopenia. JNMA J Nepal Med Assoc. 2008 Mar; 47(169):12-7.
11. Bhatnagar SK, Chandra J, Narayan S, Sharma S, Singh V, Dutta AK. Pancytopenia in children: etiological profile. J Trop Pediatr. 2005 Aug;51 (4): 236-9.

12. Khodke K, Marwah S, Buxi G, Yadav RB, Chaturvedi NK. Bone marrow examination in cases of pancytopenia. J Indian Acad Clin Med 2001JanJune. 2001;2:1-2.

13. Gupta V, Tripathi S, Tilak V, Bhatia BD. A study of clinico-haematological profiles of pancytopenia in children. Trop Doct. 2008 Oct 1;38(4):241-3.

14. Prabhala S, E J, B P, M S, T R. Bone marrow examination in pancytopenia: a study of six years. J Evol Med Dent Sci. 2014 Nov 26;3(65):14189-95.

15. Pine M, Walter AW. Pancytopenia in Hospitalized Children: A Five-year Review. J Pediatr Hematol Oncol. 2010 Jul;32(5):e192-4.

16. Khunger JM, Arulselvi S, Sharma U, Ranga S, Talib VH. Pancytopenia--a clinico haematological study of 200 cases. Indian J Pathol Microbiol. 2002 Jul;45(3):375-9.

17. N V, S D. A reappraisal of underlying pathology in adult patients presenting with pancytopenia. Trop Geogr Med. 1992 1992;44(4):322-7.

18. Azaad MAA, Li Y, Zhang Q, Wang H. Detection of Pancytopenia Associated with Clinical Manifestation and Their Final Diagnosis. Open J Blood Dis. 2015;5(3):17-30.

19. Imbert M, Scoazec JY, Mary JY, Jouzult H, Rochant H, Sultan C. Adult patients presenting with pancytopenia: a reappraisal of underlying pathology and diagnostic procedures in 213 cases. Hematol Pathol. 1989;3(4):159-67.

\section{How to cite this article?}

Yadav A, Nigam R K, Malik R. A study of clinico-hematological profile of pancytopenic patients in Central India. Int J Med Res Rev 2017;5(05):484-491. doi:10.17511/ijmrr. 2017.i05.08. 